\title{
TEMA 2016: Cáncer de Testículo: Revisión Bibliográfica
}
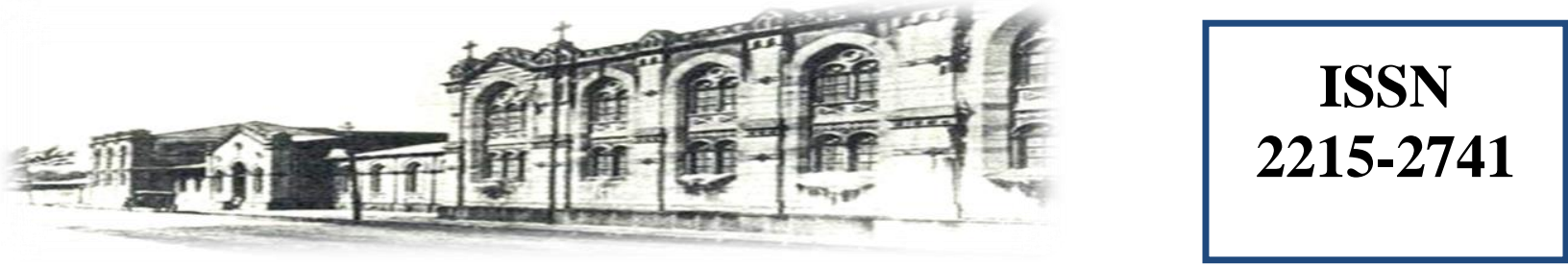

Hospital San Juan de Dios, San José, Costa Rica. Fundado en 1845

Recibido: $\quad 10 / 09 / 2015$

Aceptado: $\quad 15 / 06 / 2016$

\section{RESUMEN}

El cáncer testicular es un tipo de tumor raro que representa el $1 \%$ de los tumores malignos en los hombres. Es, sin embargo, el tipo de cáncer más común en hombres jóvenes en la población occidental. La incidencia de cáncer de testículo está aumentando en todo el mundo, a pesar de una disminución en las tasas de mortalidad evidenciadas en los países occidentales. Es importante identificar si las variaciones en las tendencias observadas entre poblaciones están ligadas a factores genéticos o ambientales.

\section{PALABRAS CLAVE}

Cáncer de testículo. Metástasis. Tumores

\begin{abstract}
Testicular cancer is a rare tumor type accounting for $1 \%$ of malignancies in men. It is, however, the most common cancer in young men in Western populations. The incidence of testicular cancer is increasing globally, although a decline in mortality rates has been reported in Western countries. It is important to identify whether the variations in trends observed between populations are linked to genetic or environmental factors.
\end{abstract}




\section{KEY WORDS}

Testicular cancer. Metastasis. Tumors.

\section{INTRODUCCIÓN}

El cáncer testicular es la neoplasia maligna más frecuente en hombres de 20 a 35 años de edad y representa aproximadamente el $1 \%$ de los tumores malignos en el género masculino. Este tumor tiene una sobrevida aproximada del $95 \%$ a los 10 años de padecerlo.

La presentación clásica suele ser una masa testicular no dolorosa en casos de tumor primario, pero esto sucede en la minoría de pacientes. En algunos casos, la tumoración testicular se acompaña de dolor difuso, endurecimiento, eritema o la combinación de alguno de estos. En pocos casos, aproximadamente en un $10 \%$ del total, puede debutar como masa palpable con dolor, esto dado posiblemente por infarto tumoral o hemorragia concomitantes. Si esto sucede puede ser confundido con Orquiepididimitis, siendo así necesaria la realización de ultrasonido si los síntomas persisten por un periodo mayor a dos semanas de tratamiento.

Estos tumores se pueden originar a partir de células Germinales (95\% de los casos) o no Germinales (5\% restante). Los tumores de células germinales tienen un alto porcentaje de curación, esto se basa en un diagnóstico y tratamiento oportunos.

Los efectos tardíos de su padecimiento y por consiguiente de su tratamiento involucran aumento del riesgo cardiovascular, neurotoxicidad, nefrotoxicidad, hipogonadismo. Existen estudios randomizados en donde se evidencia que el principal agente toxico del tratamiento es el cisplatino, el cual causa cambios en el endotelio vascular y cambios electrolítico importantes que producen mayor morbilidad a posterior.

\section{DISCUSIÓN}

Embriología del desarrollo testicular

Las gónadas aparecen inicialmente como un par de eminencias longitudinales, los pliegues $o$ crestas genitales o gonadales, que se forman por proliferación del epitelio superficial y la condensación del mesénquima subyacente. Las células germinativas primordiales solo aparecen en los pliegues genitales desde la sexta semana. Las células germinales primordiales tienen un complemento de cromosomas sexuales XY. Por influencia del gen SRY localizado en el cromosoma Y, que codifica el factor determinante testicular, los cordones sexuales primitivos siguen proliferando y se introducen profundamente en la medula gonadal para formar los cordones testiculares o medulares. Hacia el hilio de la glándula, los cordones se disgregan en una red de filamentos celulares diminutos que posteriormente darán origen a la rete testis. Durante el desarrollo ulterior, una capa compacta de tejido fibroso, la túnica albugínea, separa a los cordones del testículo de la superficie epitelial.

En el cuarto mes, los cordones testiculares toman una forma de herradura y sus extremos se continúan con los de la rete testis. Los cordones testiculares están compuestos en este momento por células germinales primordiales y células de Sertoli derivadas del epitelio superficial de la glándula. Las células intersticiales de Leydig se desarrollan a partir del mesénquima original de la cresta gonadal y se encuentran entre los cordones testiculares. Comienzan a desarrollarse poco después de iniciada la diferenciación de los cordones. En la octava semana de desarrollo, las células de Leydig empiezan a producir testosterona y el testículo puede influir entonces en la diferenciación sexual de los conductos genitales y de los genitales externos. Los cordones testiculares se mantienen íntegros hasta la pubertad, cuando se canalizan y dan origen a los túbulos seminíferos. Una vez que se ha producido su canalización, estos túbulos se unen a los de la rete testis, los cuales a su vez penetran en los conductillos eferentes. Los conductillos eferentes son las porciones remanentes de los túbulos excretores del sistema mesonéfrico y actúan como vínculo entre la rete testis y el conducto mesonéfrico o de Wolff, que recibe el nombre de conducto deferente. ${ }^{1}$

\section{Factores de Riesgo}

Criptorquidia: Termino referido al no descenso o descenso incompleto de uno o los dos testículos a través del canal inguinal hacia la bolsa escrotal. Este es el factor de riesgo más concretamente establecido para el desarrollo de cáncer testicular, elevando el riesgo de cuatro a ocho veces sobre hombres sin este antecedente. Estudios han 
demostrado que el desarrollo normal de células germinales está comprometido en pacientes con Criptorquidia.

Tumor Testicular contralateral: Un estudio cohorte realizado en Estados Unidos, que involucró a 29515 hombres reveló que los pacientes con tumor testicular contralateral tienen un riesgo 12.4 veces mayor que la población en general. Por este motivo, se recomienda realizar biopsia del testículo contralateral en casos de tumor testicular. $^{6}$

Historia familiar de cáncer testicular: Bases de datos sobre estudios acerca del cáncer testicular arrojan que los hijos de padres con antecedente de cáncer testicular tienen cuatro veces más riesgo de padecerlo, y que hermanos enfermos tienen ocho veces mayor riesgo de manifestar neoplasia testicular que la población general. Rapley et al. Reportan evidencia de un gen TGCT1, localizado en el cromosoma Xq27, que aparentemente está involucrado en la susceptibilidad para desarrollar neoplasia testicular. ${ }^{4}$

Infertilidad masculina/Disgenesia gonadal: Debido al incremento de casos de cáncer testicular en los últimos 30 años, ha existido una cantidad equitativa de descenso en la fertilidad y calidad del semen. Varios estudios realizados, han demostrado que los pacientes portadores de cáncer testicular asocian mayores tasas de infertilidad que la población general. ${ }^{4}$

Microlitiasis Testicular: Se refiere a múltiples focos hiperecoicos distribuidos difusamente por uno o ambos testículos. Su etiología es desconocida. Presenta una prevalencia de 0.6 a $6.7 \%$ en hombres asintomáticos de 18 a 35 años. ${ }^{3}$

\section{Clasificación de Tumores Testiculares}

Células Germinales: se clasifican en cinco tipos celulares básicos: seminoma, carcinoma de células embrionarias, tumor del saco vitelino, teratoma y coriocarcinoma. Más del 50\% de los TCG presentan componente mixto, sin embargo, la clasificación de estos carece de importancia en la elección del tratamiento, mientras que se resulta relevante para el momento de determinar conducta a seguir si existen metástasis de ganglios linfáticos retroperitoneales.
Seminoma: tres tipos: clásico, espermatocítico y anaplásico.

- Clásico: aproximadamente el $85 \%$ del total de seminomas. Más frecuente presente en la cuarta década de la vida. (1)

- Anaplásico: 5-10\% del porcentaje total de seminomas. Tienden a ser de peor pronóstico debido a que producen mayor cantidad de metástasis y tienen más actividad mitótica. (1)

- Espermatocítico: corresponde del 2$12 \%$ de los seminomas. La mitad se diagnostican en hombres mayores de 50 años. Producen muy pocas metástasis. (1)

Carcinoma Embrionario: lesión pequeña y redondeada, que además invade la túnica vaginal del testículo y compromete las estructuras contiguas al cordón espermático. Dentro de sus características frecuentes se encuentra el pleomorfismo, característica que les confiere un alto potencial de malignidad.

Coriocarcinoma: se puede presentar como un nódulo palpable. Los pacientes que presentan esta extirpe de tumor, pueden presentarse con metástasis a distancia avanzadas.

Teratoma: contiene más de una capa de células germinales en estadios diferentes de maduración y diferenciación. En muy pocas ocasiones, puede observarse tejido de diferenciación maligna.

Tumor del saco vitelino: es la neoplasia testicular más frecuente en lactantes y niños.

Tumores Mixtos: aproximadamente en el $60 \%$ de los pacientes portadores de tumores testiculares. La combinación más frecuente es carcinoma embrionario, tumor de saco embrionario y teratoma.

Carcinoma in situ del testículo: se conoce como el precursor preinvasor de todos los tumores de células germinales testiculares, exceptuando el seminoma espermatocítico. Se desarrolla a partir de los gonocitos fetales y en el estudio histológico se observan túbulos seminíferos que contienen sólo células de Sertoli y germinales malignas, esto dado a su pleomorfismo nuclear. Entre los factores de riesgo para desarrollar CIS testicular, se encuentra como principal al tumor de células germinales espermatocítico, otros menos relevantes como cáncer del testículo contralateral y 
criptorquidia. El principal método para detectar este tipo de tumor testicular sigue siendo la biopsia.

\section{Manifestaciones Clínicas}

Signos y síntomas: Presentación habitual del tumor testicular es un nódulo o una tumefacción indolora de una gónada. Aproximadamente un $40 \%$ de los pacientes refieren dolor sordo o sensación de pesadez en hipogastrio, región anal o escrotal. Un $10 \%$ se enteran cuando presentan metástasis, ya sea por adenopatía, tos o disnea, algunos otros como síntomas gastrointestinales (anorexia, náuseas, vómitos o hemorragia).

\section{Estudios por imágenes}

Radiografía de tórax: En primer lugar deben realizarse proyecciones anteroposterior $\mathrm{y} d \mathrm{de}$ lateral. $^{8}$

Tomografía Axial Computarizada: Es el estudio más efectivo para detectar compromiso de ganglios linfáticos retroperitoneales. Ha venido reemplazando con el tiempo, debido a su precisión a la urografía intravenosa y linfangiografía. ${ }^{8}$

\section{Marcadores Tumorales}

Alfafetoproteina: En la formación embriológica del feto, es producida por el saco vitelino, aparato digestivo e hígado. Sus concentraciones más elevadas se encuentran durante la semana 12 de gestación, y disminuyen un año después del nacimiento. Los valores normales en hombres son de menos de $40 \mathrm{mcg} / \mathrm{L}$. Es producida por el carcinoma embrionario puro, el teratocarcionma, el tumor del saco vitelino o por la combinación de estos. ${ }^{12}$

Gonadotrofina coriónica humana: En condiciones normales, es producida por el tejido trofoblástico. En los TCG, las células sincitiotrofoblásticas son las que producen HCG, cuya vida media es de 24 a 36 horas. El rango normal para los hombres es de 0-5 UI/ml. Todos los pacientes con coriocarcinoma y más de la mitad de quienes presentan Carcinoma Embrionario elevan este marcador tumoral. ${ }^{12}$
Deshidrogenasa Láctica: Es detectada en musculo liso, cardiaco, esquelético, hígado, riñón y cerebro. Sus valores normales oscilan de 105 a 333 UI/L. Es útil en detección y seguimiento de tumores de células germinales, sin embargo, deben excluirse otras entidades que estén produciendo elevación de esta enzima. ${ }^{12}$

Fosfatasa alcalina: existe poca evidencia sobre su uso en detección de tumores testiculares, sin embargo se ha demostrado que su elevación radica en casos de enfermedad avanzada. Los valores normales en hombres son de 80-306 UI/L. Dentro de los diagnósticos diferenciales, lo elevan Hepatitis, anemia, alcoholismo, enfermedades óseas, entre otros. ${ }^{12}$

Con respecto a la aplicación clínica de los marcadores tumorales, del 50 a $70 \%$ de los pacientes con tumores testiculares no seminomatosos presenta niveles elevados de Alfafetoproteina. ${ }^{1}$

\section{Fundamentos terapéuticos}

Tumores de células germinales: El primer paso en el tratamiento de pacientes en los que se sospecha una neoplasia testicular es la orquiectomía radical con ligadura alta del cordón espermático. Se prefiere orquiectomía inguinal como método para establecer control local de la enfermedad. El factor principal en la evolución natural de las neoplasias testiculares es el diagnóstico histológico. Posterior a la orquiectomía podría considerarse como opción terapéutica a seguir la Radioterapia. $^{5}$

Cuando se trata de tumores confinados a un solo testículo menores a dos centímetros se elige la cirugía conservadora. El tratamiento hacia los pacientes que presentan metástasis a distancia en principio es la quimioterapia. La disección de ganglios linfáticos retroperitoneales es una excelente opción para estadificar la extensión de los tumores testiculares y además eliminar siembras de la neoplasia, posterior a esto, se ha demostrado que la quimioterapia aumenta la sobrevida en los pacientes afectados. ${ }^{10}$

\section{Tumores Secundarios de Testículo}

Linfoma: Corresponden al 5\% del total de tumores testiculares, siendo el secundario más fre- 
cuente confinado a este órgano y el más frecuente en pacientes mayores de 50 años.

Infiltración leucémica del testículo: Es un sitio importante de recidiva en niños con LLA.

\section{CONCLUSIONES}

El cáncer de testículo sigue teniendo baja prevalencia en la población general, sin embargo tiene cada vez mayor incidencia en la mayoría de países; a pesar de esto, las tasas de mortalidad siguen siendo bajas y la mayoría de los hombres se curan. La comprensión de los riesgos y efectos secundarios a largo plazo del tratamiento son importantes en el manejo de los hombres con esta enfermedad.

Es importante enfatizar a la población masculina en la realización del autoexamen testicular, y ante la presencia de alguna masa sospechosa en escroto, en especial en pacientes con factores de riesgo, acudir a la consulta urológica.

\section{BIBLIOGRAFÍA}

1. Alan Wein et al. Campbell-Walsh Urology. $10^{\text {th }}$ Edition. Editorial Elsevier. P. 837-899.

2. B. Mester, T. Behrens, S. Dreger. (2010). Occupational Causes of Testicular Cancer in Adults. Bremen Institute for Prevention Research and Social Medicine (BIPS). University of Bremen, Bremen, Germany. P. 160-170.

3. DeCastro B, Peterson A, Costabile R. A five-year followup study of asymptomatic men with testicular microlithiasis. $\mathrm{J}$ Urol 2008; 179: 1420-1423

4. F. K. Mostof. Testicular Tumors, Epidemiologic, Etiologic, and Pathologic Features. Forces Institute of Pathology, Washington, D.C. P. 1186-1201.

5. Lois B. Travis, Clair Beard, James M. (2010) Testicular Cancer Survivorship: research Strategies and recommendations. Rubin Center for Cancer Survivorship and Department of Radiation Oncology.

6. Planelles J, Beltrán JR, Tarín M, (2007). Cancer testicular bilateral: presentación de cuatro casos. Hospital Universi- tario Dr. Peset. Valencia. Actas Urol Española; 31(10):1117-1122.

7. Rustom P. Manecksha and John M. Fitzpatrick. (2009). Epidemiology of testicular cancer. University Hospital, Dublin, Ireland. P. 1329-1333.

8. Saavedra J, Ramirez C, Peña G. (2009). Cáncer de testículo. Anales de Radiología, México. 1:47-59.

9. Shanmugalingam et al (2013) Clinical Epidemiology. King's College London, School of Medicine, Division of Cancer Studies 2013:5 417-427.

10. Valderrama-Gómez RA, CondoriSaldaña J, Claros-Gutierrez PG, ClarosMatienzo CA. Cáncer testicular con metástasis. Rev Méd-Cient "Luz Vida". 2011; 2(1):76- 80.

11. Valsero H, María E; Samaniego $P$. (2012). Cáncer testicular, nuestra experiencia en diez años. Archivos Españoles de Urología, vol. 65. p. 467-475.

12. Magdelénat H. Tumor markers in oncology: past, present and future. J Immunol Methods 1992; 150:133-143

\section{CONFLICTO DE INTERÉS Y/O AGRADE- CIMIENTOS}

Los autores declaran que no existió ningún conflicto de interés en el presente reporte. 